\title{
A ASSISTÊNCIA ONCOLÓGICA NO SISTEMA ÚNICO DE SAÚDE: O CASO DO CÂNCER DE MAMA NO NORTE DE MINAS GERAIS
}

Sandra Célia Muniz Magalhães Doutora em Geografia - UFU sandramunizgeo@hotmail.com

Mônica Oliveira Alves Doutoranda do Programa de Pós Graduação em Geografia do Instituto de Estudos Socioambientais da Universidade Federal de Goiás - PPGEO/IESA/UFG monicaelit@hotmail.com

Flávia de Oliveira Santos Doutora em Geografia - UFU flavia.santos@ufu.br

\section{RESUMO}

O presente trabalho tem como objetivo discutir a regionalização e a organização dos serviços de saúde para assistência ao usuário com câncer de mama no Norte de Minas Gerais. Os procedimentos metodológicos utilizados consistiram em pesquisa bibliográfica e documental, observação in loco, além da utilização de dados disponibilizados pelo Instituto Brasileiro de Geografia e Estatística e pelo Instituto Nacional do Câncer. Os resultados obtidos evidenciaram o esforço existente por parte do sistema público em parceria com o setor privado para melhorar a assistência oncológica na região. Contudo, os recursos disponíveis, tanto os tecnológicos, como humanos ou estruturais, ainda são poucos e ineficientes para atenderem à demanda, gerando imensas filas nos dias de atendimento e demora na marcação de consultas e exames. Nota-se, ainda, que no Norte de Minas Gerais, a assistência oncológica está concentrada em uma única cidade dentro da Região Ampliada de Saúde, sendo necessária a descentralização desses serviços para melhor atender à demanda por tratamento da doença. Ressalta-se que quem luta contra o câncer luta também contra o tempo, principal opositor para uma possível cura da doença.

Palavras-chave: Regionalização da saúde. Assistência Oncológica. Câncer de mama.

\section{ONCOLOGICAL ASSISTANCE IN THE SINGLE HEALTH SYSTEM: THE CASE OF BREAST CANCER IN NORTH OF MINAS GERAIS}

\begin{abstract}
The present study aims to discuss the regionalization and organization of health services to assist the user with breast cancer in the North of Minas Gerais. The methodological procedures used consisted of bibliographic and documentary research, in situ observation, besides the use of data made available by the Brazilian Institute of Geography and Statistics and the National Cancer Institute. The results obtained evidenced the efforts made by the public system in partnership with the private sector to improve oncological care in the region. However, the available resources, technological as well as human or structural, are still few and inefficient to meet the demand, generating huge queues in the days of attendance and delay in the appointment of consultations and exams. It is also noted that in the North of Minas Gerais, cancer care is concentrated in a single city within the Expanded Health Region, and it is necessary to decentralize these services to better meet the demand for treatment of the disease. It is emphasized that those who fight against cancer also fight against time, the main opponent for a possible cure of the disease.
\end{abstract}

Keywords: Regionalization of health. Oncological Assistance. Breast cancer. 


\title{
INTRODUÇÃO
}

Sabe-se que as neoplasias malignas, em especial o câncer de mama, representam um grave problema de saúde pública no Brasil. Este fato que se reflete também na escala dos estados e municípios, sendo necessária a intervenção dos órgãos de governo, de todas as esferas, no planejamento e organização do sistema de saúde, a fim de se promover o acesso de forma universal e equitativo, garantindo a saúde como um bem público e um direito individual e coletivo aos usuários, principalmente aos adoecidos por câncer (RÊGO; NERY, 2013).

Para se garantir o acesso pleno e equitativo da população afetada pelo câncer aos serviços de saúde, deve haver um planejamento adequado, que considere "[...] a identificação dos pólos de atração, a regionalização do atendimento, as distâncias percorridas pela população na busca pela assistência, e os volumes envolvidos nestes deslocamentos" (OLIVEIRA et al., 2011, p. 318). Devese enfatizar que dentre os princípios gerais da Política Nacional para Prevenção e Controle do Câncer - PNPCC estão a organização, regionalização e descentralização das redes de atenção, respeitando-se os critérios de acesso, escala e escopo (BRASIL, 2013).

Uma das formas que vêm sendo utilizadas na tentativa de se assegurar o acesso pleno aos serviços de saúde no Brasil é a regionalização deste setor, através do Plano Diretor de Regionalização - PDR. O PDR é um instrumento de planejamento e gestão, utilizado na área da saúde, com o objetivo de descentralizar a oferta dos serviços, proporcionando aos usuários uma maior e mais adequada acessibilidade, considerados os princípios da integralidade, equidade e economia de escala. Nesse contexto, a Região é vista como:

\begin{abstract}
[...] conjunto de municípios circunvizinhos que mantêm entre si inter-relações para as questões de promoção e assistência à saúde do SUS, sendo a maioria periféricos a um polo que exerce força de atração sobre os demais, e constituem-se como base populacional e territorial para cálculos do planejamento em saúde e organização de redes. Para o PDR/MG, cada região se configura conforme o modelo de atenção adotado; cada região se caracteriza segundo o nível de atenção, o âmbito de cobertura dado, o porte, as inter-relações com as demais regiões do sistema, circunvizinhas ou não. (MINAS GERAIS, 2011, p. 29)
\end{abstract}

O Brasil possui grandes dimensões territoriais, com acentuadas desigualdades socioeconômicas entre suas regiões. Tal fato reflete diretamente no acesso à saúde, principalmente da população mais pobre. O mesmo ocorre dentro dos estados, como é o caso de Minas Gerais, com um território vasto e com características sociais, econômicas e culturais diversas. Daí a relevância de se promoverem estudos de cunho regional na tentativa de evidenciar as potencialidades e fragilidades que as regiões apresentam, para que assim as políticas públicas de saúde possam ser desenvolvidas como maior eficácia, facilitando o acesso da população aos serviços de saúde.

Diante do exposto, o objetivo deste trabalho é discutir a regionalização e a organização dos serviços de saúde para assistência ao usuário com câncer de mama no Norte de Minas Gerais. Os procedimentos metodológicos utilizados consistiram em pesquisa bibliográfica e documental, observação in loco, além da utilização de dados disponibilizados pelo Instituto Brasileiro de Geografia e Estatística e pelo Instituto Nacional do Câncer. A partir do Sistema de Informação Geográfica (SIG) os dados obtidos foram utilizados no processamento digital das imagens utilizadas no trabalho, gerando novas informações referentes à organização dos serviços de saúde na região em estudo. Foi utilizado o software ArcGiz 10.2 e a base cartográfica do estado de Minas Gerais disponibilizadas pelo IBGE. Assim, foram editados os mapas utilizados no trabalho, como resultado do processamento das informações.

\section{A REGIONALIZAÇÃO DA SAÚDE NO NORTE DE MINAS GERAIS}

É inquestionável que o câncer, em geral, e o de mama em particular, se apresenta como um grave problema de saúde pública no Brasil, o que reflete também nos estados e municípios, sendo necessária a intervenção dos órgãos de governo, de todas as esferas, no planejamento e organização do sistema de saúde, a fim de se promover o acesso de forma universal e equitativo, garantindo a saúde como um bem público e um direito individual e coletivo (RÊGO; NERY, 2013).

Para se garantir o acesso pleno e equitativo da população afetada pelo câncer aos serviços de saúde, deve haver um planejamento adequado, que considere "[...] a identificação dos pólos de atração, a regionalização do atendimento, as distâncias percorridas pela população na busca pela assistência, e os volumes envolvidos nestes deslocamentos" (OLIVEIRA et al., 2011, p. 318). Deve-

$\begin{array}{llllll}\text { Caminhos de Geografia } \quad \text { Uberlândia-MG } & \text { v. 21, n. } 75 & \text { Jun/2020 } & \text { p. 284-299 Página } 285\end{array}$


se enfatizar que dentre os princípios gerais da PNPCC estão a organização, regionalização e descentralização das redes de atenção, respeitando-se os critérios de acesso, escala e escopo (BRASIL, 2013).

Uma das formas que vêm sendo utilizadas na tentativa de se assegurar o acesso pleno aos serviços de saúde no Brasil é a regionalização deste setor, através do Plano Diretor de Regionalização (PDR). O PDR é um instrumento de planejamento e gestão, utilizado na área da saúde, com o objetivo de descentralizar a oferta dos serviços, proporcionando aos usuários uma maior e mais adequada acessibilidade, considerados os princípios da integralidade, equidade e economia de escala. Nesse contexto, a Região é vista como:

[...] conjunto de municípios circunvizinhos que mantêm entre si inter-relações para as questões de promoção e assistência à saúde do SUS, sendo a maioria periféricos a um polo que exerce força de atração sobre os demais, e constituem-se como base populacional e territorial para cálculos do planejamento em saúde e organização de redes. Para o PDR/MG, cada região se configura conforme o modelo de atenção adotado; cada região se caracteriza segundo o nível de atenção, o âmbito de cobertura dado, o porte, as inter-relações com as demais regiões do sistema, circunvizinhas ou não. (MINAS GERAIS, 2011, p. 29).

O Brasil possui grandes dimensões territoriais, com acentuadas desigualdades socioeconômicas entre suas regiões. Tal fato reflete diretamente no acesso à saúde, principalmente da população mais pobre. O mesmo ocorre dentro dos estados, como é o caso de Minas Gerais, com um território vasto e com características sociais, econômicas e culturais diversas. Daí a relevância de se promoverem estudos de cunho regional na tentativa de evidenciar as potencialidades e fragilidades que as regiões apresentam, para que assim as políticas públicas de saúde possam ser desenvolvidas como maior eficácia, facilitando o acesso da população aos serviços de saúde.

O Plano Diretor de Regionalização da Saúde de Minas Gerais - PDR/MG foi instituído no ano de 2002, tendo em vista a distribuição e o ordenamento dos espaços territoriais do Estado, visando à organização dos serviços de saúde em redes, de forma a garantir os princípios da equidade, integralidade, economia de escala, escopo e acessibilidade da população. O documento foi elaborado em conformação com as diretrizes estabelecidas pela Norma Operacional da Assistência à Saúde NOAS/SUS 01/02 que estabelece, entre outros, o processo de regionalização como estratégia de hierarquização e equidade dos serviços de saúde.

A NOAS/SUS 01/02 institui o PDR como instrumento de ordenamento do processo de regionalização da assistência no âmbito dos Estados e no Distrito Federal. Estes deverão organizar seus territórios em regiões/microrregiões de saúde e módulos assistenciais, com a identificação dos municípios sede, municípios polo e dos demais municípios abrangidos. A elaboração do PDR deve ser realizada de forma a garantir o acesso dos cidadãos a um conjunto de ações e serviços vinculados a responsabilidades mínimas, o mais próximo possível de sua residência, como o acompanhamento de pessoas com doenças crônicas de alta prevalência, entre as quais está o câncer (BRASIL, 2002).

O PDR/MG foi construído de forma a incorporar a construção social de redes de atenção à saúde e considerou o fluxo da população mineira em busca desses serviços no Estado. Essas redes devem articular os pontos de atenção à saúde em territórios sanitários, assegurando uma oferta de serviços mais eficiente, efetiva e com qualidade. Assim, os serviços de atenção primária à saúde devem ser ofertados no âmbito dos municípios, o mais próximo das residências. As unidades secundárias que ofertam serviços de média complexidade devem estar concentradas nas microrregiões (Regiões de Saúde). Enquanto que as unidades terciárias, cujos serviços englobam a alta complexidade, concentram-se nas macrorregiões ou Regiões Ampliadas de Saúde (MINAS GERAIS, 2011). A regionalização utilizada no PDR/MG segue um modelo de atenção à saúde composto por três níveis:

MUNICÍPIO - Base territorial de planejamento da atenção primária à saúde, sendo preferencialmente organizada e ofertada por meio de equipes de PSF.

MICRORREGIÃO DE SAÚDE - Base territorial de planejamento da atenção secundária à saúde, com capacidade de oferta de serviços ambulatoriais e hospitalares de média complexidade e, excepcionalmente, de alguns serviços de Alta Complexidade (AC), caso da Terapia Renal Substitutiva (TRS). Deve ser constituída por um conjunto de municípios contíguos, com população de cerca de 
150.000 habitantes que estão adscritos a um município de maior porte (município polo) com oferta mais ampla do elenco proposto para o nível micro.

MACRORREGIÃO DE SAÚDE - Base territorial de planejamento da atenção terciária à saúde que engloba microrregiões de saúde (e seus municípios) em função da possibilidade de oferta e acesso a serviços de saúde ambulatoriais e hospitalares de maior densidade tecnológica: a Alta Complexidade (AC) nos termos da Tabela do Ministério da Saúde (MS) - SIA e SIH. Deve ainda ter capacidade de oferta de Procedimentos de Média Complexidade Especial. Esses procedimentos, embora da $M C$, requerem maior tecnologia, sua oferta é rarefeita no Estado e a demanda requer agregação, ou seja, formação de escala. A abrangência deve ser macrorregional, para uma população em torno de 1.500 .000 habitantes.

O município polo é caracterizado como aquele que exerce força de atração sobre outros, em número comparativamente significativo, por sua capacidade atual e potencial de equipamentos urbanos e de fixação de recursos humanos especializados. (MINAS GERAIS, 2011, 63-64).

As regiões mineiras apresentam características ${ }^{1}$ divergentes entre si, tanto no que se refere à distribuição populacional, quanto nos aspectos socioeconômicos, ambientais e culturais, fato que acarreta dificuldades ao se implantar um PDR. A região Norte de Minas, por exemplo, apresenta uma grande extensão territorial, com um contingente populacional relativamente pequeno, o que caracteriza um quadro de pequena densidade demográfica e de grandes distâncias entre seus municípios (MINAS GERAIS, 2011). Pode-se afirmar que o Norte de Minas é uma região dual, onde podem ser encontrados espaços de riqueza e de pobreza, tradicionais e modernos, de produção e escassez, de discursos e de realidades (PEREIRA, 2007). A região, ora assinalada como 'bolsão de pobreza', ora como 'cheia de potencialidades', como afirma Pereira e Soares (2005), diferencia-se das demais regiões mineiras, tanto pelas características ambientais marcadas, principalmente, pelos impactos do clima semi-árido, como pelas características socioeconômicas, uma vez que apresenta graves problemas sociais em âmbito regional. As autoras enfatizam que:

O Norte de Minas é um espaço que se individualiza no contexto estadual pela pobreza, pela seca, pela marginalização, pelo isolamento regional, pela dependência dos municípios frente às transferências da União e do estado, fenômenos que, historicamente, aproximam mais essa região do Nordeste brasileiro do que do Sudeste (PEREIRA; SOARES, 2005, p.11609).

É interessante ressaltar que a região Norte de Minas foi pioneira na implantação da Rede de Urgência e Emergência no Estado, com base em uma metodologia preconizada no protocolo de Manchester em 1997. De acordo com Magalhães (2013), isto se deu pelo fato de a região necessitar de urgentes intervenções no setor de saúde por apresentar diversas evidências epidemiológicas, bem como deficiência no registro de mortalidade, muitas vezes apontada como sendo de causas mal definidas, o que revela certa fragilidade na assistência à saúde na região, entre outros. Apesar dos notáveis progressos, a autora aponta vários entraves que dificultam a qualidade dos serviços ofertados na região, como a escassez de leitos e de recursos humanos nas unidades de saúde e as longas distâncias existentes entre os centros que ofertam tais serviços e os demais municípios que compõe a região, e destaca a necessidade de melhoria quantitativa e qualitativa em muitos quesitos, principalmente em recursos humanos:

No Norte de Minas existe um serviço estruturado, porém ainda está aquém da demanda. Observa-se que o sistema de saúde e as necessidades da população parecem andar na contramão, já que existe uma estrutura de atendimento em determinados municípios, é disponibilizada uma infinidade de novas estratégias de atenção à saúde, enquanto uma multidão de indivíduos não tem acesso a eles (MAGALHÃES, 2013, p. 120).

\footnotetext{
${ }^{1}$ Sobre a região Norte de Minas, em todos os seus aspectos, inclusive sobre a organização do sistema de saúde, sugere-se consultar os trabalhos de Pereira (2007) Cidade Média e Região: o significado de Montes Claros no Norte de Minas Gerais e Magalhães (2013) Fatores Determinantes da Ocorrência de Tuberculose no Norte de Minas Gerais.
}

$\begin{array}{llllll}\text { Caminhos de Geografia } & \text { Uberlândia-MG } & \text { v. 21, n. } 75 & \text { Jun/2020 } & \text { p. 284-299 } & \text { Página } 287\end{array}$


O território mineiro, que está dividido em 13 Regiões Ampliadas de Saúde, com 76 Regiões de Saúde. A RAS Norte de Minas é a maior região do estado mineiro em extensão territorial e contém a terceira maior população do Estado (MINAS GERAIS, 2014). A figura 01 apresenta a localização da RAS Norte de Minas e o município Montes Claros, considerado polo regional no que se refere à oferta de serviços.

Figura 01 - Mapa de localização da RAS Norte de Minas.

(12)

Observa-se pela figura 01, que o município de Montes Claros ocupa uma posição geográfica que o favorece como polo regional, uma vez que oferece diversos serviços, em vários setores, como saúde e educação, atraindo para si a população dos outros municípios da região.

Como já mencionado, o Estado de Minas Gerais possui uma grande extensão territorial com 586.528,293 km2, dividido em 853 municípios. Sua população total, de acordo com o Censo Demográfico do IBGE em 2010, foi de 19.597.330 habitantes, estimada em 20.869.101 para 2015, com uma densidade demográfica de $33,42 \mathrm{hab} / \mathrm{km}^{2}$ (IBGE, 2015). A RAS Norte de Minas abrange 86 municípios que estão divididos em 9 Regiões de Saúde (Fig. 02), com uma população total de aproximadamente 1.661.130 habitantes, sendo Montes Claros a cidade polo que oferece os serviços ambulatoriais e hospitalares de alta complexidade (MINAS GERAIS, 2011).

Montes Claros é o polo regional, oferecendo os mais variados serviços como educação, saúde, serviços jurídicos, entre outros; e comércio diversificado atendendo a toda região norte mineira e, inclusive municípios de outros estados, como os do sul da Bahia. Dentre todos esses serviços, a cidade tem destaque importante no setor de saúde, por oferecer vários serviços médicos, com especialidades ímpares, tendo uma rede hospitalar interligada com diversas clínicas médicas, sendo referência regional (PEREIRA, 2007) no tratamento de diversas doenças, principalmente aquelas que dependem dos serviços de alta complexidade, como é o caso do câncer. 


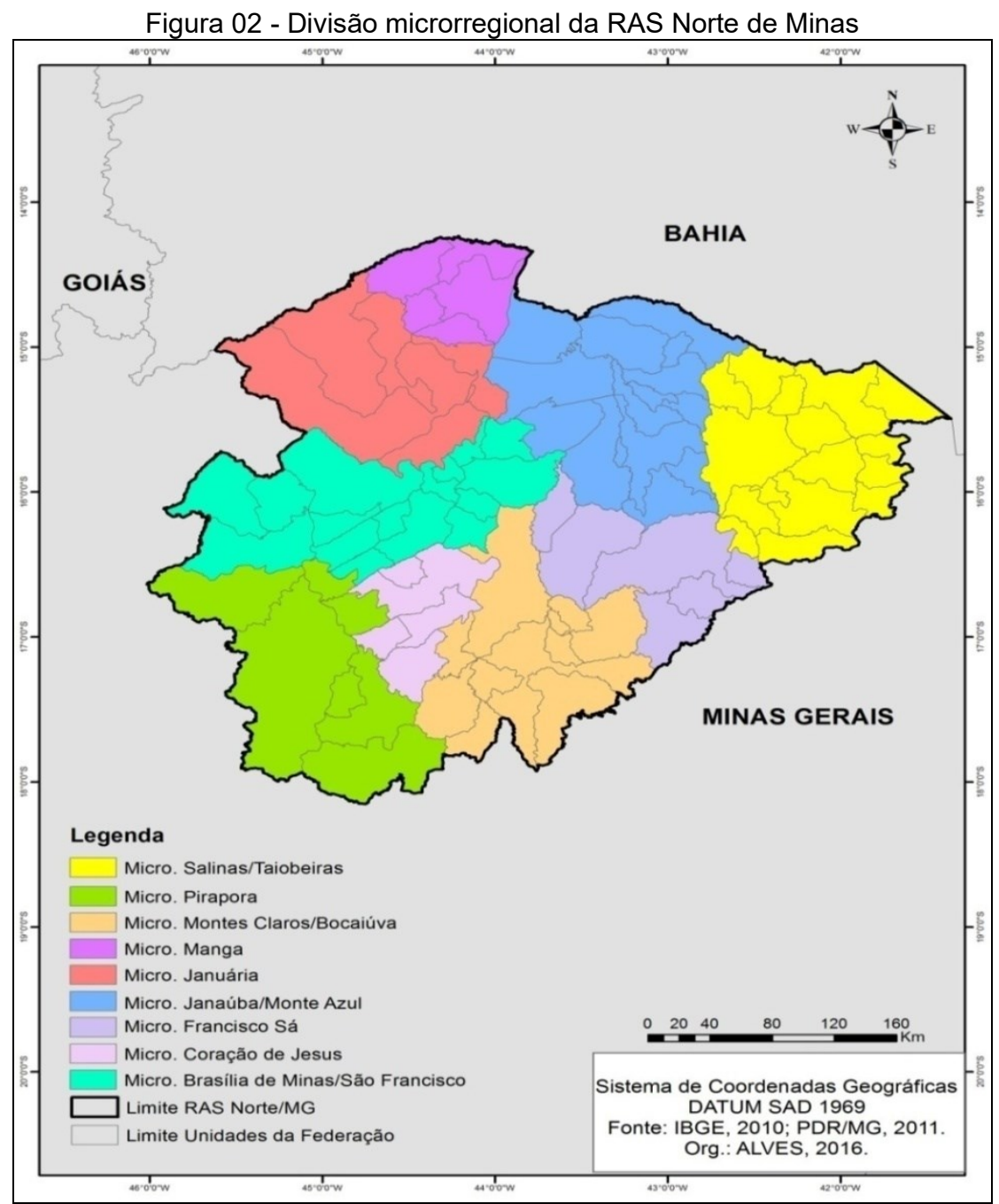

Os atendimentos realizados no âmbito da Rede de Atenção Oncológica não estão restritos à população dos municípios que compõem a região da qual a unidade hospitalar que oferece os serviços faz parte, e os usuários devem buscar o atendimento o mais próximo possível de sua residência. Dessa forma, um grande número de pessoas com neoplasia maligna, inclusive com câncer de mama, converge para a cidade de Montes Claros, em busca de tratamento para a doença, causando uma sobrecarga nos hospitais que prestam o atendimento, o que dificulta o acesso dessa população aos serviços de saúde, como demora na marcação de exames, nos procedimentos ambulatoriais, nas cirurgias, entre outros. Em se tratando de câncer, são indispensáveis a rapidez e a agilidade no atendimento para a tentativa de cura, pois muitos tipos da doença apresentam um bom prognóstico se diagnosticados e tratados oportunamente, como é o caso do câncer de mama.

\section{A ASSISTÊNCIA AO USUÁRIO COM CÂNCER DE MAMA NO NORTE DE MINAS GERAIS}

A organização de um atendimento integral, humanizado e hierarquizado é fator primordial na assistência oncológica, dada a complexidade que o tratamento para a doença requer. Deve-se lembrar de que o câncer de mama apresenta um prognóstico relativamente bom, se diagnosticado e tratado precocemente. As altas taxas de mortalidade pela doença no Brasil se dão pelo estágio avançado em que a doença é diagnosticada, fazendo-se necessária a constituição de uma linha de cuidado que vá desde as ações de rastreamento aos cuidados paliativos, envolvendo todos os níveis de atenção à saúde. De acordo com Peters (2013, p. 39), uma linha de cuidado eficaz "[...] garantirá um fluxo ágil no atendimento com ações de referência e contra referência implementadas com 
relações interpessoais, estabelecidas com contato estreito entre os diversos setores e a responsabilização pelos casos em todos os níveis assistenciais".

A assistência oncológica no âmbito do SUS é organizada para atender de forma integral e integrada os usuários que buscam pelo tratamento do câncer. Essa assistência é assegurada por meio de uma Rede de Atenção Oncológica, planejada, organizada e controlada pelas Secretarias de Saúde estaduais e municipais, que têm como objetivo adequar a prevenção e o tratamento do câncer às necessidades de cada região do país, segundo os níveis de atenção à saúde. Assim, cada nível de atenção possui as atribuições que Ihes são pertinentes. A prevenção primária em Oncologia consiste em ações que visam à redução da exposição da população aos fatores de risco para a doença. Já a prevenção secundária, refere-se ao rastreamento da doença através de exames mais específicos (CESTARI; ZAGO, 2005). No quadro 01, é possível visualizar todas as fases da abordagem do câncer de mama.

Quadro 01 - Fases da abordagem do câncer de mama.

\begin{tabular}{l|l}
\hline \multicolumn{1}{c|}{ FASE } & \multicolumn{1}{c}{ PROCEDIMENTOS } \\
\hline Exame Clínico das Mamas - ECM & $\begin{array}{l}\text { Realizado em UBS por profissional que desenvolve a atenção } \\
\text { primária. }\end{array}$ \\
\hline Exame Mamográfico - MMG & $\begin{array}{l}\text { Realizado na atenção secundária, por profissional médico } \\
\text { treinado, em mulheres com exames clínicos alterados. }\end{array}$ \\
\hline Exame Ultrassonográfico - UGS & $\begin{array}{l}\text { Também deve ser realizado na atenção secundária, por } \\
\text { profissional médico treinado, em mulheres com exames clínicos } \\
\text { alterados. }\end{array}$ \\
\hline Biópsia & $\begin{array}{l}\text { Realizado na atenção secundária, por profissional médico } \\
\text { treinado, em mulheres com exames clínicos alterados, a partir de } \\
\text { procedimentos como a punção aspirativa por agulha fina (PAAF), } \\
\text { ou punção por agulha grossa (core biopsy), quando indicadas. }\end{array}$ \\
\hline Tratamento & $\begin{array}{l}\text { Uma vez que é diagnosticado o câncer de mama, a mulher é } \\
\text { encaminhada às unidades de atenção terciária (CACON e e } \\
\text { UNACON), para que o tratamento adequado seja instituído. }\end{array}$ \\
\hline
\end{tabular}

Fonte: PETERS, 2013.

Org.: ALVES, 2016.

$\mathrm{Na}$ atenção primária, realizada no âmbito da Unidade Básica de Saúde (UBS), devem ser desenvolvidas as ações de promoção da saúde, com incentivo a adoção de hábitos de vida saudáveis (dieta adequada, atividade física, combate ao tabagismo e ao alcoolismo e à obesidade) e a conscientização da importância da prevenção e do diagnóstico precoce do câncer de mama em todas as etapas do rastreamento. Na UBS devem ser realizados os exames clínicos das mamas, conforme quadro 01 , solicitação dos exames mamográficos na população de risco, encaminhamento dos casos de resultados mamográficos com alguma evidência de alteração, entre outros (PETERS, 2013).

A atenção secundária se refere à unidade de Média Complexidade do SUS e deve ser unidades ambulatoriais especializadas (regionais ou municipais). Sua função é dar seguimento aos casos de exames alterados, sendo necessária a presença de profissionais capacitados para realizarem as intervenções diagnósticas nos casos suspeitos de câncer de mama, como mamografia, biópsia por agulha grossa, punção por agulha fina, e o tratamento de lesões benignas mamárias (PETERS, 2013).

A atenção terciária é composta por estabelecimentos habilitados como Unidade de Assistência de Alta Complexidade em Oncologia/UNACON ou Centro de Assistência de Alta Complexidade em Oncologia/CACON. As unidades hospitalares assim classificadas devem oferecer assistência especializada e integral ao usuário com câncer, abrangendo as seguintes modalidades integradas: diagnóstico, cirurgia oncológica, radioterapia, quimioterapia (oncologia clínica, hematologia e oncologia pediátrica), medidas de suporte, reabilitação e cuidados paliativos (BRASIL, 2010). 
É responsabilidade das UNACONs realizar o diagnóstico definitivo e o tratamento dos cânceres mais prevalentes da região de saúde onde está inserido, oferecendo minimamente os tratamentos de cirurgia e quimioterapia, e devem ainda, obrigatoriamente, ter o tratamento de radioterapia referenciado e contratualizado formalmente. Os CACONs devem realizar o diagnóstico definitivo e o tratamento de todos os tipos de câncer, mas não obrigatoriamente dos cânceres raros e infantis. Também devem realizar tratamento de cirurgia, radioterapia e quimioterapia dentro de sua estrutura hospitalar. O Estado de Minas Gerais possui três CACONs, dois na capital Belo Horizonte e um na cidade de Muriaé, e vinte e nove UNACONs (MINAS GERAIS, 2012). Através da figura 03, pode-se observar como estão espacializados os CACONs/ UNACONs no território mineiro.

Figura 03 - Espacialização dos CACONs/ UNACONs em Minas Gerais.

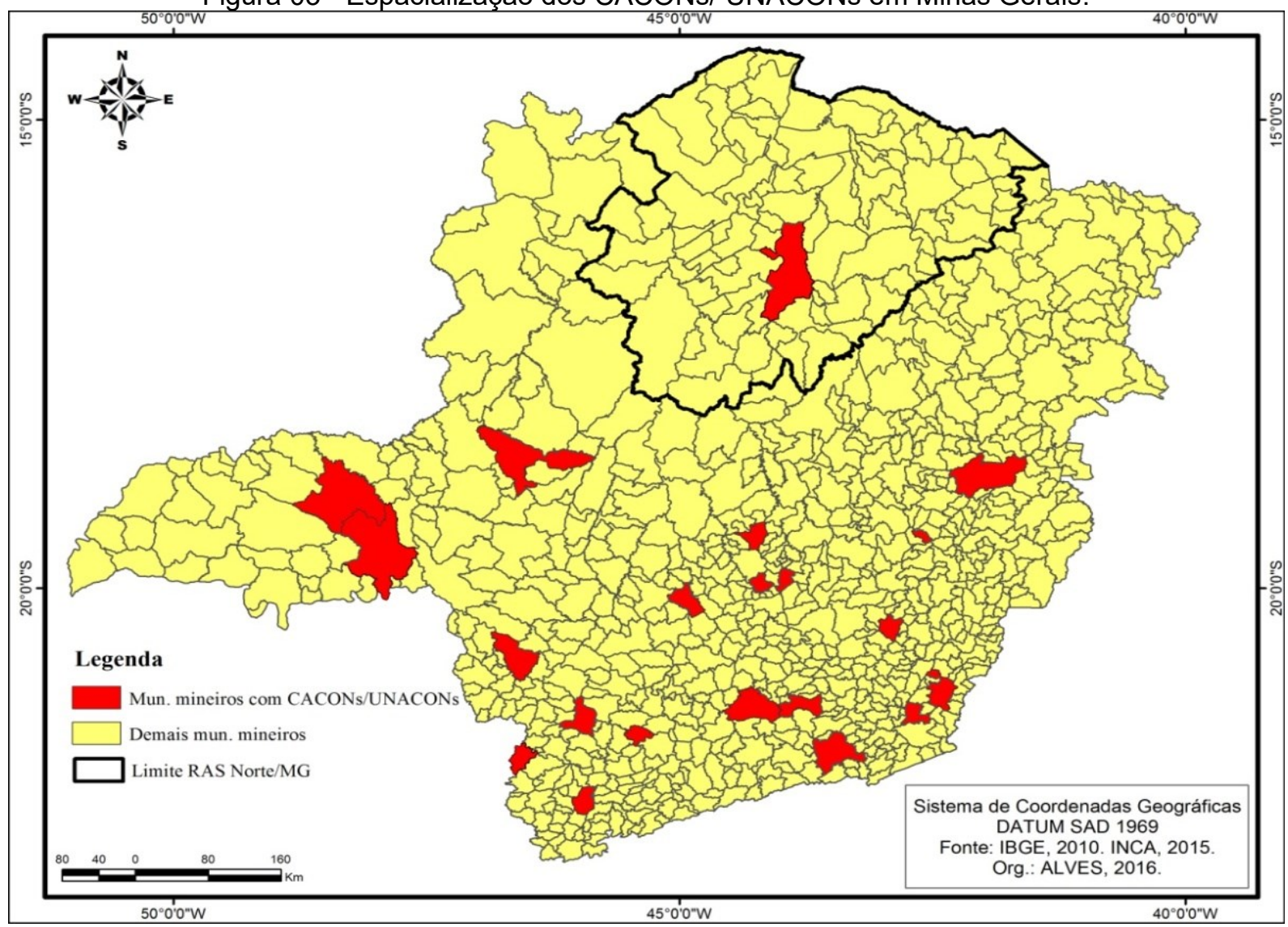

Nota-se, pela figura 03 , que as desigualdades regionais, que são características marcantes no território mineiro, refletem-se principalmente no setor de saúde, havendo nas regiões centrais e sul uma maior disponibilidade de serviços, equipamentos e recursos humanos especializados. Observase que as unidades hospitalares que oferecem os serviços de alta complexidade oncológica concentram-se na porção sul do Estado de Minas Gerais, enquanto que, ao norte, somente o município de Montes Claros disponibiliza tais serviços. Na RAS Norte de Minas, a cidade de Montes Claros, considerada cidade polo, comporta a assistência hospitalar e ambulatorial referentes à atenção oncológica na região, a partir de dois hospitais consideradas UNACONs, a Fundação de Saúde Dilson de Quadros Godinho e Irmandade Nossa Senhora das Mercês (Santa Casa de Montes Claros) (Fig. 04). 
Figura 04 - Fachada das unidades de atendimento oncológico em Montes Claros/MG.

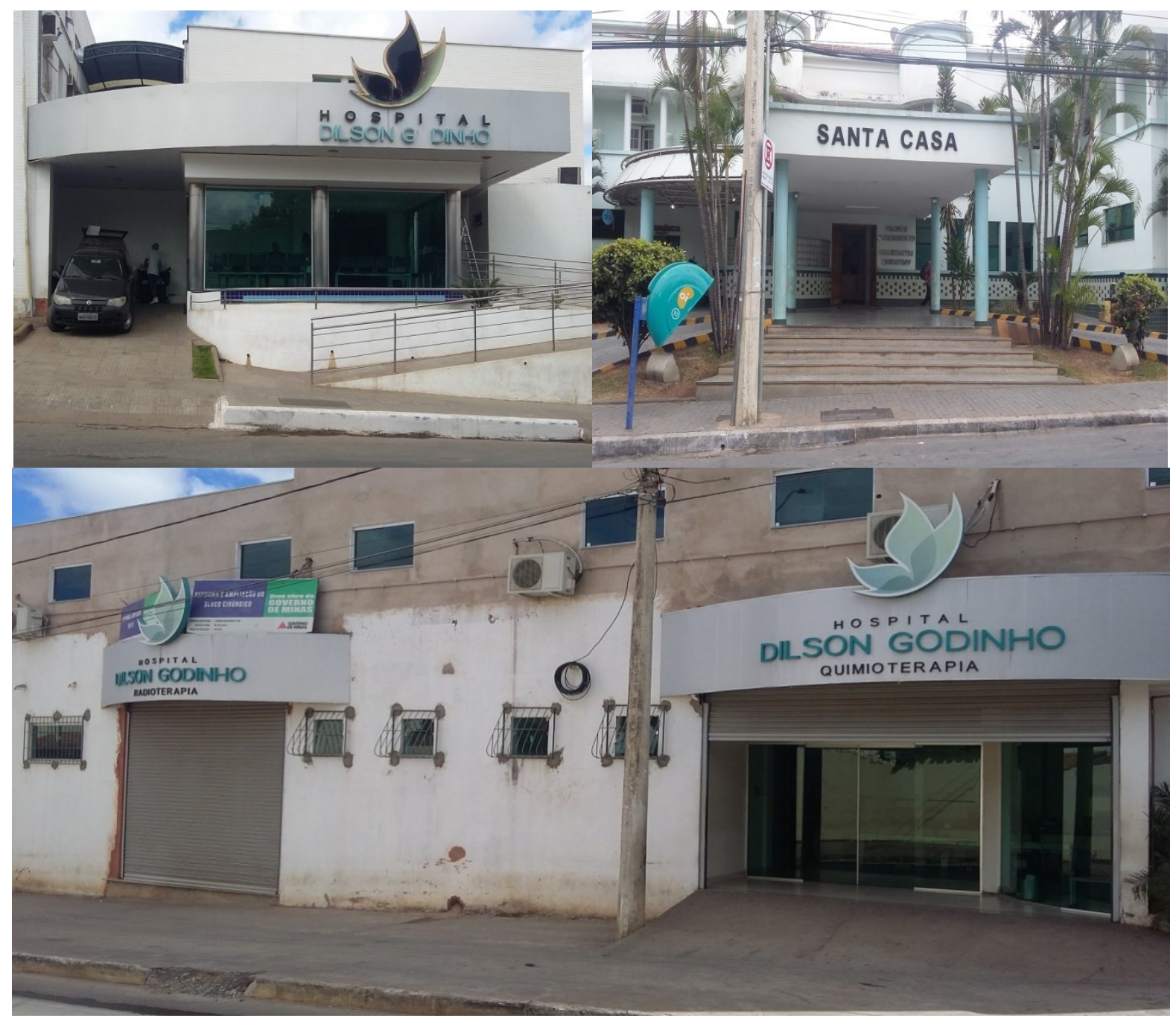

Autor: ALVES, 2016.

Estas duas unidades de saúde recebem toda a demanda de usuários com câncer, inclusive o de mama, da RAS Norte de Minas Gerais e de outras regiões, tanto de Minas Gerais, como de outros estados, como do sul da Bahia. Pela figura 04, é possível perceber que os setores de quimioterapia e radioterapia do Dílson Godinho ainda se encontram em construção, apesar de já realizarem os procedimentos necessários para o tratamento do câncer. Em dias de atendimento, é possível perceber o grande número de pessoas, doentes ou acompanhantes, que buscam pelo tratamento nas duas unidades, lembrando que muitos vêm todos os dias de outros municípios para Montes Claros. Contudo, observou-se que, entre o grupo de mulheres que participaram da pesquisa, 13 dessas afirmaram ter começado o tratamento pela doença dentro de dois meses após o diagnóstico, como é preconizado pela Lei dos Sessenta Dias. Outras seis começaram a se tratar até seis meses após o diagnóstico.

Nota-se que há um esforço na tentativa de parcerias do sistema público com o setor privado, como preconizado pela PNPCC, para melhorar a assistência oncológica na região. Contudo, os recursos disponíveis, tanto os tecnológicos, como humanos ou estruturais, ainda são poucos e ineficientes para atenderem à demanda, gerando imensas filas nos dias de atendimento e demora na marcação de consultas e exames. Ressalte-se que quem luta contra o câncer luta também contra o tempo, principal opositor para uma possível cura da doença.

A informação em saúde para a população e para os usuários potenciais dos serviços constitui-se numa relevante característica para a melhoria do acesso à saúde. O conhecimento sobre o sistema e os serviços de saúde, sobre a doença e suas alternativas terapêuticas, contribui para facilitar a

Caminhos de Geografia Uberlândia-MG $\quad$ v. 21, n. $75 \quad$ Jun/2020 $\quad$ p. 284-299 Página 292


utilização dos serviços. Deve-se destacar que este conhecimento envolve, além das ações desenvolvidas pelo setor de saúde, o capital cultural das famílias, o nível de escolaridade, o acesso aos meios de informação, o que pode resultar em desigualdades sociais no acesso (TRAVASSOS; CASTRO, 2012).

É possível analisar o acesso ao conhecimento da população da RAS Norte de Minas através do IDHM Educação (Fig. 05).

Figura 05 - Municípios da RAS Norte/MG - IDHM Educação, 2010.

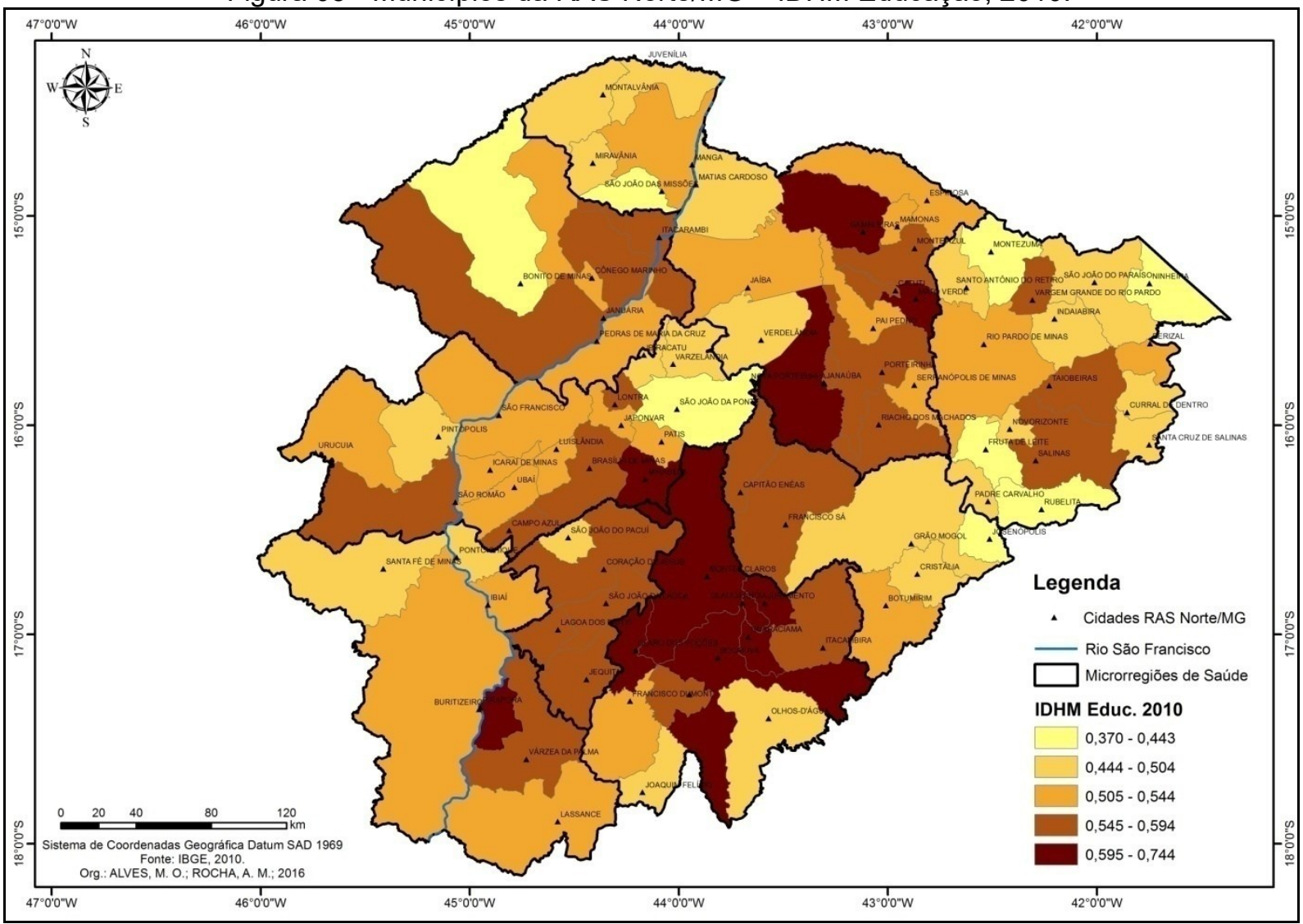

O IDHM Educação mede o acesso da população ao conhecimento através de duas variáveis, a escolaridade da população adulta e o fluxo escolar da população jovem. A partir da análise da figura 05, é possível observar que a maior parte dos municípios da RAS Norte/MG apresentou um índice educacional classificado como baixo (48 municípios) e muito baixo (26 municípios), em 2010. Entre os cinco municípios que apresentaram o IDHM Educação muito baixo, estão Fruta de Leite, São João das Missões, Bonito de Minas, Ninheira e São João da Ponte, com os índices 0,370, 0,381, 0,388, 0,418 e 0,419 , respectivamente. Entre os cinco melhores classificados, destaca-se Montes Claros, único município da região com índice educacional considerado alto, seguido por Pirapora, Glaucilândia, Guaraciama, Janaúba e Bocaiúva, com índices de 0,680, 0,667, 0,655, 0,649 e 0,644, respectivamente, considerados como índices médios.

No caso de Montes Claros, não é surpresa a sua colocação, já que o município desponta como polo educacional da região. Contudo, deve-se salientar que o município de Guaraciama, que se encontra entre os melhores classificados em termos de acesso ao conhecimento, é o município que apresentou a maior incidência da doença, podendo estar o nível de instrução de sua população diretamente relacionada com o aumento nos registros de câncer de mama no município. Outro fator a se considerar é a proximidade de Guaraciama com o centro especializado na assistência oncológica, no caso de Montes Claros, fator primordial para o maior acesso da população aos serviços de saúde, assim como o acesso à informação, que está relacionado com o nível de escolaridade (Gráfico 01).

$\begin{array}{llllll}\text { Caminhos de Geografia } & \text { Uberlândia-MG } & \text { v. 21, n. } 75 & \text { Jun/2020 } & \text { p. 284-299 Página } 293\end{array}$


Gráfico 01 - Nível de escolaridade das usuárias participantes da pesquisa.

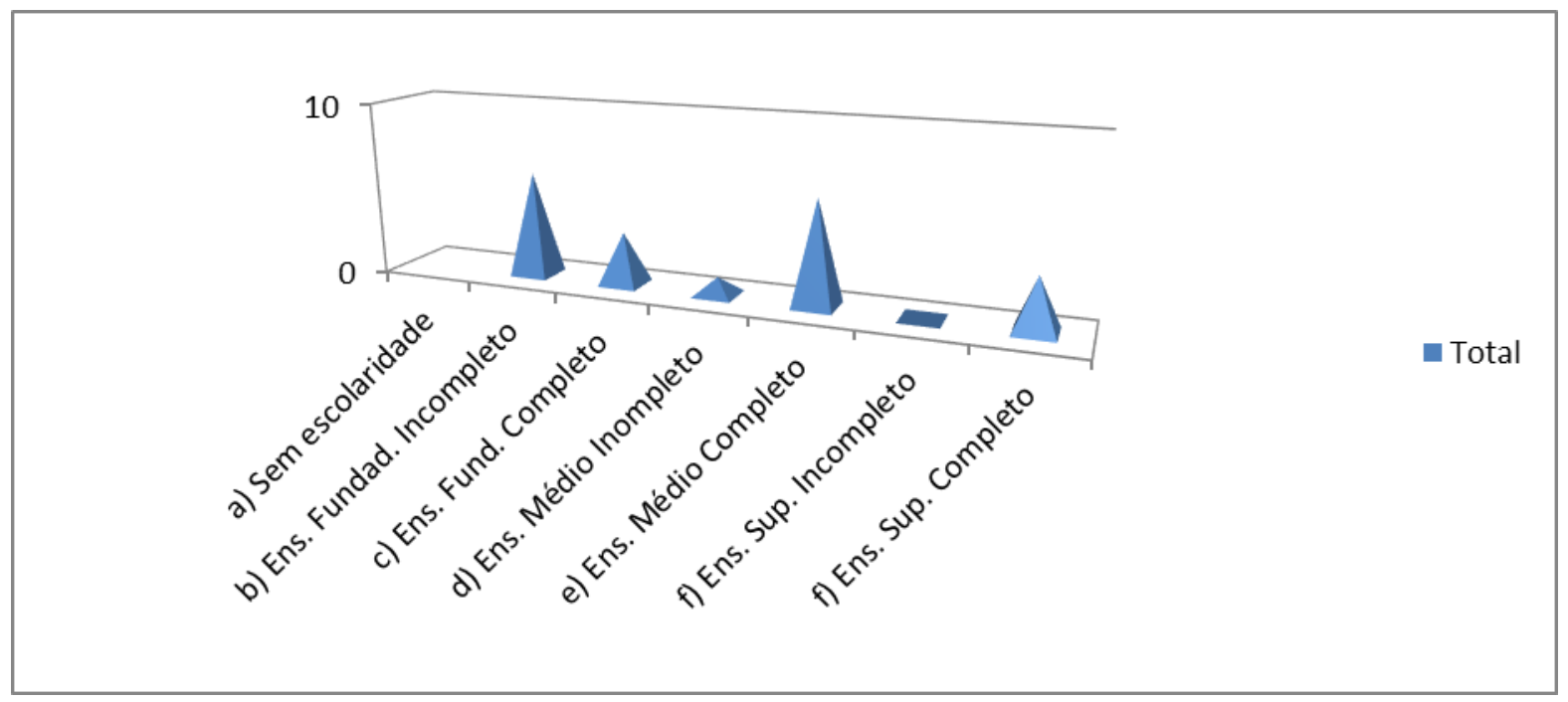

Fonte: Coleta direta, 2016.

Org.: ALVES, 2016.

É possível observar, através do gráfico 01, que o nível de escolaridade das mulheres participantes da pesquisa, apresentou-se bem variado e relativamente satisfatório, já que boa parte dessas possuía Ensino Médio e Superior completos, o que corrobora com o fato de que quanto maior a escolaridade, maior o nível de conhecimento e de informação, e maior o acesso ao sistema de saúde.

Quanto aos meios de acesso à informação, praticamente todas consideram a televisão como principal provedora, como mostra o gráfico 02.

Gráfico 02: Principal forma de acesso à informação das usuárias participantes da pesquisa.

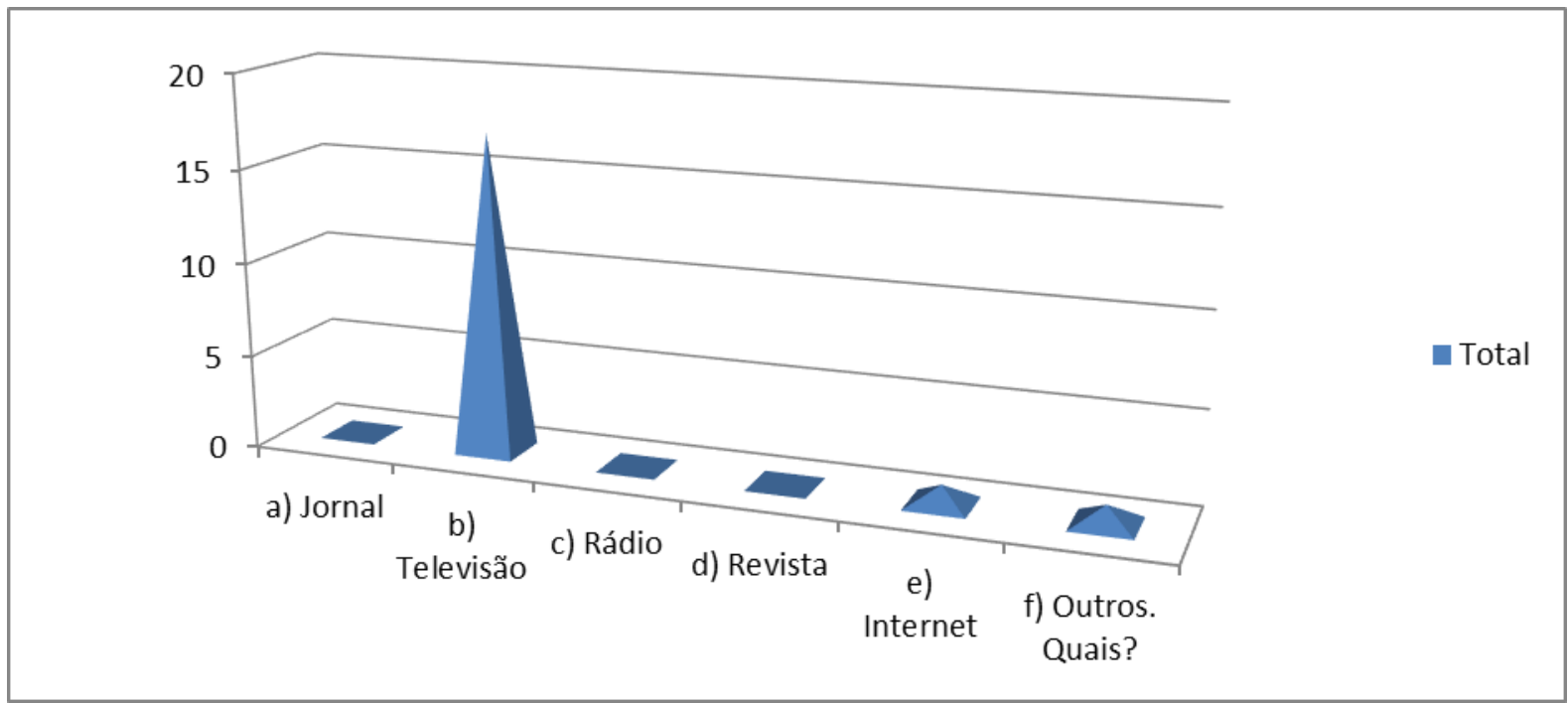

Fonte: Coleta direta, 2016.

Org.: ALVES, 2016.

A maior parte das participantes elencou a televisão como sendo sua principal forma de acesso ao conhecimento, como demonstrado no gráfico 02 , inclusive as mulheres residentes na zona rural.

$\begin{array}{llllll}\text { Caminhos de Geografia } \quad \text { Uberlândia-MG } & \text { v. 21, n. } 75 & \text { Jun/2020 } & \text { p. 284-299 Página } 294\end{array}$


Ressalta-se aqui a importância dos meios midiáticos, principalmente os televisivos, na divulgação de informações que visem à prevenção de doenças, como o câncer de mama, e a promoção da saúde.

Outra forma de levar informação à população sobre a importância da prevenção e diagnóstico precoce do câncer de mama são as ações e eventos realizados por entidades filantrópicas, em parceria com o setor público e privado. Algumas dessas ações puderam ser acompanhadas em Montes Claros, no dia 17 de abril de 2015. Trata-se da $5^{\mathrm{a}}$ edição do Mutirão de Prevenção ao Câncer. O evento, que ocorre anualmente, é organizado pela Associação Presente de Apoio ao Paciente com Câncer Padre Tiãozinho, em parceria com o Hospital Santa Casa de Montes Claros, a Prefeitura Municipal de Montes Claros através da Secretaria Municipal de Saúde, empresas do ramo farmacêutico, laboratórios, profissionais da saúde, acadêmicos, entre outros.

De acordo com a coordenadora do Mutirão, a Associação Presente é uma instituição filantrópica, como já citada anteriormente, fundada, no ano de 2004, pelo Padre Tiãozinho e pela oncologista e presidente da Associação, Priscila Bernardina Miranda Soares. Tem como objetivo assistir às pessoas carentes vitimadas pelo câncer na região, em todos os quesitos, como atendimentos psicológicos, assistência social, fornecimento de cesta-básica e medicamentos, hospedagem, transporte, alimentação e atividades de lazer. Também realiza ações de rastreamento precoce da doença, como é o caso do Mutirão. Segundo a coordenadora, o padre Tiãozinho adoeceu com câncer e foi assistido pela Presidente da Associação. Em pleno tratamento, o padre observou as dificuldades vivenciadas pelas pessoas vítimas da doença esperando por tratamento nos hospitais da cidade. A partir daí, surgiu a ideia da criação da instituição.

O evento compõe o calendário de ações sociais da cidade e tem como objetivo diagnosticar e orientar a comunidade sobre o câncer, para que a população se conscientize da importância da realização de exames regulares, assim como da mudança de hábitos e comportamentos, a fim de se prevenir a doença e promover a saúde coletivamente. O Mutirão ocorre todos os anos na Praça Dr. Carlos Versiane, centro de Montes Claros. De acordo com a coordenadora, a escolha do local se deu por esta praça estar situada em um ponto central, onde há um grande fluxo de pessoas, oriundas de várias partes da cidade (Fig. 06).

Figura 06 - 5ª edição do Mutirão de Prevenção ao Câncer Montes Claros/MG - 2015.

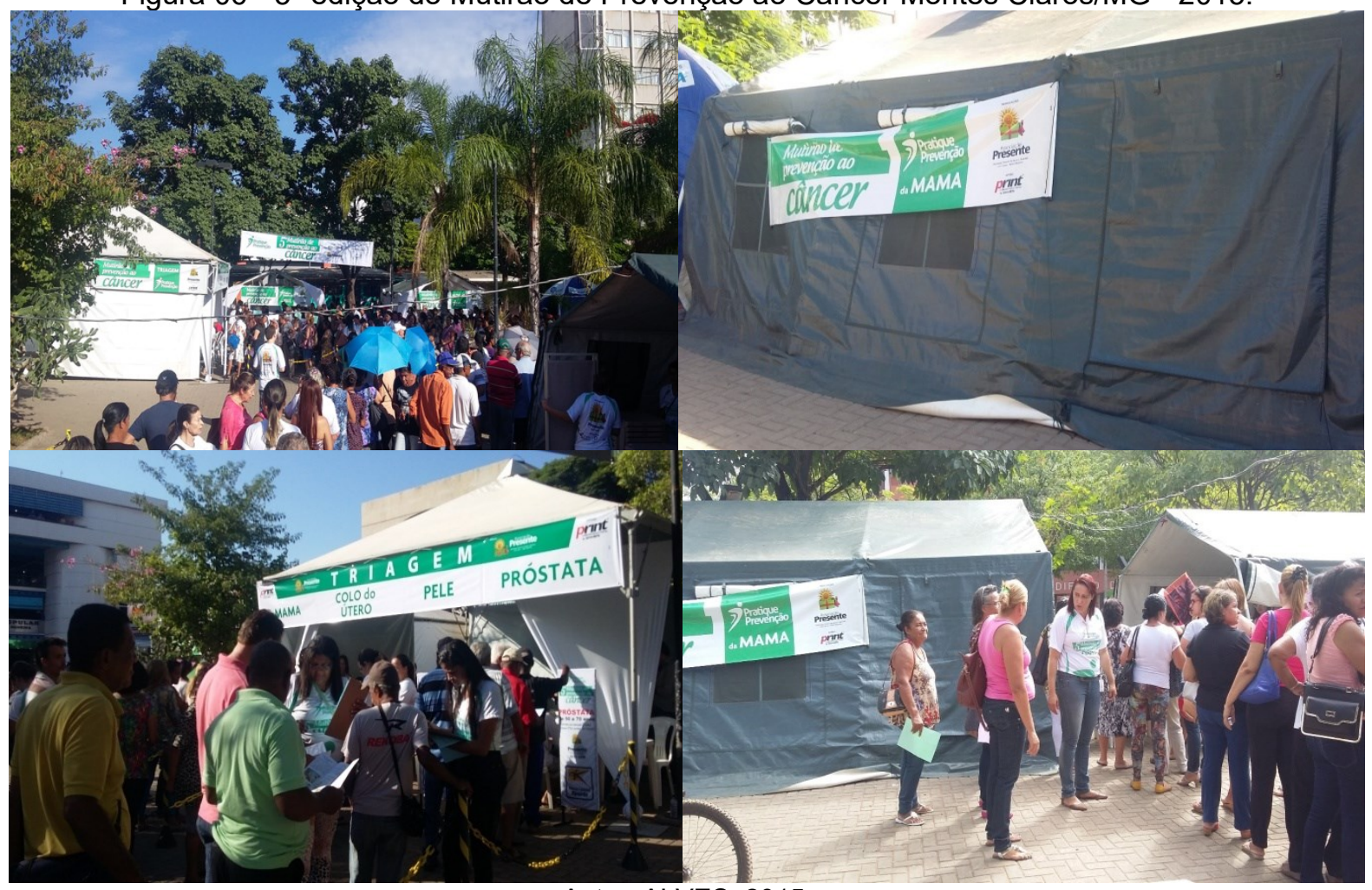

Autor: ALVES, 2015. 
Para a realização dos atendimentos, foram preparadas oito tendas consultórios (Fig. 06), em que trabalharam um grupo com cerca de 200 voluntários, entre os quais estavam médicos especialistas como oncologistas, radioterapeutas, mastologistas, urologistas, dermatologistas, ginecologistas, odontólogos, assim como enfermeiros, nutricionistas e acadêmicos. Foram realizados exames clínicos e laboratoriais, bem como fornecidas orientações nutricionais gratuitas à população na tentativa prevenir e/ou diagnosticar casos de câncer de mama, boca, pele, colo uterino e próstata, que estão entre os tipos de câncer mais recorrentes na macrorregião Norte de Minas. Foram realizados 1687 atendimentos em mais de 10 horas de ocorrência do evento. De acordo com informações disponíveis na página da Associação Presente, na $4^{a}$ edição (2014), o evento registrou mais de 1800 atendimentos, com 39 diagnósticos positivos para o câncer. Nessa $5^{a}$ edição do Mutirão, houve um número recorde de participantes do sexo masculino em busca de exames preventivos para o câncer de próstata, como afirmou a coordenadora do evento. Este fato demonstra a importância de ações educativas para a prevenção de doenças e promoção da saúde.

Em relação ao câncer de mama, as participantes primeiramente realizavam o exame clínico com mastologistas. As mulheres que estavam dentro da faixa etária de maior risco para o desenvolvimento da doença, no caso de 50 a 69 anos, todas eram encaminhadas para a realização da mamografia. Quanto às outras participantes, caso houvesse suspeita de tumores na mama após o exame clínico, também eram conduzidas para agendarem as mamografias. No dia do evento, foram agendadas mais de 80 mamografias, que seriam realizadas pela Santa Casa de Montes Claros. Em conversa com algumas mulheres participantes do evento, todas foram enfáticas ao afirmar que o evento é muito importante, principalmente no que se refere à marcação de consulta e exames, em especial a mamografia, pois existe muita dificuldade em se conseguir marcá-los normalmente nas UBS. Uma das mulheres afirmou que, apesar de ter conseguido fazer uma mamografia, não conseguiu agendar com nenhum médico para análise do resultado do exame. Outra afirmou que foi detectado um nódulo na mama pelo exame clínico e, embora com o pedido da mamografia em mãos, ainda não havia conseguido agendar o exame.

Muitas outras ações de prevenção ao câncer de mama e promoção da saúde são realizadas na cidade de Montes Claros, principalmente no mês de outubro, eleito como mês de prevenção à doença, o chamado "Outubro Rosa". Não obstante, percebe-se que essas ações não envolvem toda a população da região em estudo, principalmente aquelas pessoas mais carentes econômico, social e culturalmente, residentes nos municípios mais longínquos em relação a Montes Claros, muitas inclusive, moradoras da zona rural. Daí a necessidade de se estender essas ações, através de projetos de extensão, em parceria das universidades com os hospitais, prefeituras, UBS, empresas privadas e a população em geral, a fim de levar informação a toda população da região, na tentativa de se garantir um acesso mais equitativo aos serviços de saúde.

Dentre as principais queixas apresentadas por essas mulheres estão as dificuldades financeiras agravadas pela doença, os efeitos da quimioterapia, as longas distâncias percorridas em busca do tratamento, a falta de informação sobre a doença e sobre o tratamento, ter que deixar de realizar suas atividades diárias, entre outros. Sobre seus medos, afirmaram receio de metástases, da retirada da mama, da queda do cabelo, dos preconceitos e, é claro, do óbito. Aprenderam, com o advento da doença, a valorizar mais a vida e a buscar mais conhecimento. Destacaram a importância de ações de prevenção não só no mês de outubro, mas em todo tempo e em todos os municípios. Afirmaram ter encontrado apoio para superar a doença nas suas famílias e na religião. Todas elogiaram o atendimento dos profissionais da saúde das unidades de assistência oncológica em Montes Claros.

Diante da situação epidêmica do câncer de mama no Brasil, como problema de saúde pública, as ações visando à prevenção da doença e à promoção da saúde da população tornam-se de extrema relevância. É sabido que, para se conseguir prevenir o câncer, é necessária a máxima redução ou eliminação dos agentes carcinogênicos, considerando os fatores ambientais, econômicos, sociais e culturais. Diante deste contexto,

Para prevenir o câncer a população deve ser informada sobre os comportamentos de risco, os sinais de alerta e a freqüência da prevenção. Mas, além disto, é importante a capacitação dos recursos humanos que atuam nesta área, buscando uma reorientação para a cultura do câncer e consequentemente mudanças na práxis destes profissionais. (CESTARI; ZAGO, 2005, p. 220) 
Percebe-se que, nos últimos anos, vem aumentando o conhecimento da população acerca das formas de prevenção de doenças potencialmente letais como o câncer, bem como a conscientização da necessidade de mudança nos hábitos e estilo de vida a fim de se alcançar uma situação de saúde plena. Cada vez mais, as ações e estratégias com foco na prevenção e promoção de saúde, através da apropriação do conhecimento sobre doenças, riscos e saúde, estão sendo disponibilizadas à população, principalmente através dos meios de comunicação de massa, como é o caso da televisão. Contudo, essas práticas estão aquém de atingir toda a demanda populacional para realmente controlar o câncer. Prova disto é o fato de que existe ainda um grande número de mortes pela doença no Brasil e no mundo. Pôde-se observar, no decorrer da pesquisa, que a maior parte dos usuários não conhece os direitos legais que os amparam, o que impede a reivindicação do direito à saúde como portadora de câncer de mama.

O acesso ao tratamento oncológico em um país com grandes dimensões territoriais, distribuição desigual da população e dos serviços de saúde, como o Brasil, apresenta graves problemas, como as ofertas desiguais de serviços e fragilidade na estrutura da rede assistencial. Sabe-se que a demora no acesso e na resolutividade contribui consideravelmente para a progressão da doença. Para o controle e tratamento do câncer de mama e de qualquer tipo de neoplasia maligna, torna-se necessário organizar toda a rede de atenção à saúde, na tentativa de melhorar o acesso dos usuários portadores da doença ao tratamento, assim como facilitar o acesso das mulheres ao diagnóstico precoce do câncer de mama através do rastreamento mamográfico. Este é um desafio enfrentado pelo SUS, que vem tentando garanti-lo através de políticas públicas de saúde específicas como a Política Nacional para a Prevenção e Controle do Câncer.

\section{CONSIDERAÇÕES FINAIS}

Diante da importância epidemiológica do câncer e a sua magnitude como problema de saúde pública, várias ações e programas vêm sendo realizados no Brasil, na tentativa de se prevenir e controlar a doença através do diagnóstico precoce, e de se garantir melhores condições de acesso da população doente aos serviços de saúde. Entre as principais políticas desenvolvidas neste âmbito, tem destaque a Política Nacional para a Prevenção e Controle do Câncer, instituída no Brasil no ano de 2013, com o objetivo de identificar e intervir sobre os determinantes e condicionantes dos diversos tipos de câncer e desenvolver ações intersetoriais de responsabilidade pública e da sociedade civil para promoção da saúde e a qualidade de vida.

Para o bom desempenho das políticas públicas de saúde foi adotado no Brasil um modelo de regionalização desse setor que consiste em uma estratégia para o planejamento adequado da oferta e distribuição dos serviços de saúde. O Plano Diretor de Regionalização da Saúde é um instrumento de fundamental importância para gestar e direcionar a descentralização desses serviços, promovendo maior e mais adequado acesso dos usuários no âmbito das regiões. No Brasil, diversas dificuldades podem ser encontradas ao se implantar o PDR, uma vez que, seja qual for a escala de análise, as regiões do país apresentam desigualdades entre si e em seu interior. É o caso do Estado de Minas Gerais, que possui uma grande extensão territorial, e suas regiões diferem tanto na distribuição populacional, quanto nos aspectos socioeconômicos, políticos, culturais, ambientais e na oferta de serviços de saúde.

O acesso ao tratamento oncológico no Norte de Minas apresenta graves problemas como as ofertas desiguais de serviços e fragilidades na estrutura da rede assistencial. Sendo assim, para o melhor controle e tratamento mais efetivo do câncer de mama, faz-se necessário reorganizar a oferta dos serviços em oncologia, descentralizando os tratamentos mais usuais, como os radioterápicos e quimioterápicos, ao nível das Regiões de Saúde, para amenizar as dificuldades de acesso ao tratamento, principalmente da população mais pobre. É necessário ainda, que se equipem as unidades de referência para os serviços oncológicos com recursos humanos e tecnológicos mínimos para a realização dos exames diagnósticos e do tratamento, como as biópsias, as cirurgias, as terapias químicas e as terapias por radiação. Todas as considerações tecidas acima se colocam como desafios enfrentados pelo SUS, que vem tentando superá-los através de políticas públicas de saúde específicas como a Política Nacional para a Prevenção e Controle do Câncer. 


\section{AGRADECIMENTOS}

O presente trabalho foi realizado com apoio: Da Coordenação de Aperfeiçoamento de Pessoal de Nível Superior - Brasil (CAPES) - Código de Financiamento 001; Do Conselho Nacional de Pesquisa CNPq; Da Fundação de Amparo à Pesquisa do Estado de Minas Gerais - FAPEMIG

\section{REFERÊNCIAS}

BRASIL. Ministério da Saúde. Portaria $n^{\circ} 874$, de 16 de maio de 2013. Institui a Política Nacional para a Prevenção e Controle do Câncer na Rede de Atenção à Saúde das Pessoas com Doenças Crônicas no âmbito do Sistema Único de Saúde (SUS). Brasília, 2013. Disponível em: http://bvsms.saude.gov.br/bvs/saudelegis/gm/2013/prt0874 1605 2013.html . Acesso em 28 de maio de 2015.

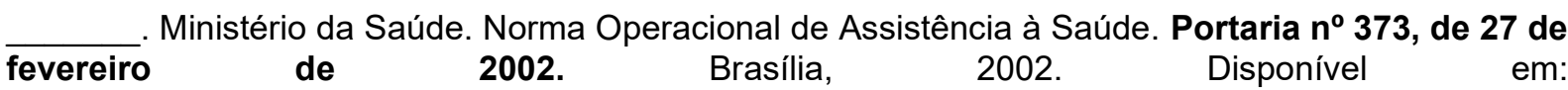
http://bvsms.saude.gov.br/bvs/saudelegis/gm/2002/prt0373 2702 2002.html . Acesso em 25 de abril de 2015.

Ministério da Saúde. Plano de Ações Estratégicas Para o Enfrentamento das Doenças Crônicas Não Transmissíveis (DCNT) no Brasil 2011-2022. Brasília - DF. 2010. Disponível em: http://bvsms.saude.gov.br/bvs/publicacoes/plano acoes enfrent dent 2011.pdf . Acesso em 27 de abril de 2015 .

CESTARI, M. E. W; ZAGO, M. M. F. A prevenção do câncer e a promoção de saúde: um desafio para o Século XXI. Rev. Bras. Enferm. 2005; mar-abr; v. 58, no 2, p. 218-21. https://doi.org/10.1590/S0034-71672005000200018

INSTITUTO BRASILEIRO DE GEOGRAFIA E ESTATÍSTICA. Diretoria de Pesquisa. Coordenação de Trabalho e Rendimento. Pesquisa Nacional Por Amostra de Domicílios Contínua - PNADC. 2015. Disponível em:

http://www.ibge.gov.br/home/estatistica/indicadores/trabalhoerendimento/pnad continua/analise02.sht

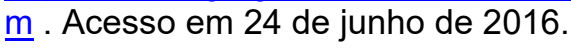

MAGALHÃES, S. C. M. Fatores determinantes da ocorrência de tuberculose no Norte de Minas Gerais. 225 f. Tese (Doutorado em Geografia). Uberlândia. Universidade Federal de Uberlândia UFU. Instituto de Geografia, Programa de Pós-Graduação em Geografia, 2013.

MINAS GERAIS. Secretaria de Estado de Saúde. Deliberação CIB-SUS/MG no 1.115, de 18 de abril de 2012. Aprova a prestação de serviços de definição de diagnostico e início de tratamento do Câncer de Mama nas Unidades de Assistência de Alta Complexidade em Oncologia/UNACON, Centros de Assistência de Alta Complexidade em Oncologia/CACON e Centros de Referência de Alta Complexidade em Oncologia. Belo Horizonte, 2012. Disponível em: http://saude.mg.gov.br/sobre/institucional/deliberacao-cib-susmg?start=40 . Acesso em 10 de outubro de 2015.

$\begin{array}{cc}\text { SER/MG, } & \text { Secretaria de Estado de Saúde. Plano Diretor de Regionalização. Belo Horizonte: } \\ \text { em: } & \text { Disponível }\end{array}$ http://www.saude.mg.gov.br/images/documentos/Livro\%20Plano\%20Diretor\%20de\%20Regionalizaca o\%20-\%20ultima\%20versao.pdf . Acesso em 25 de abril de 2015.

Secretaria de Estado da Saúde. Programa de Avaliação e Vigilância do Câncer e seus

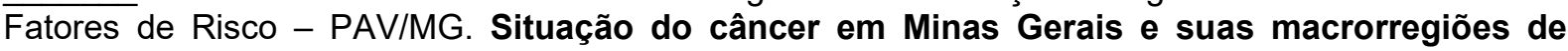
saúde: estimativas de incidência e mortalidade para o ano 2013, válidas para 2014: perfil da mortalidade: perfil da assistência na alta complexidade / Secretaria de Estado da Saúde de Minas Gerais. - Belo Horizonte: SES-MG, 2014. v. 1.

CESTARI, M. E. W; ZAGO, M. M. F. A prevenção do câncer e a promoção de saúde: um desafio para o Século XXI. Rev. Bras. Enferm. 2005; mar-abr; v. 58, n² 2, p. 218-21. 
OlIVEIRA, E. X. G. de.; MELO, E. C. P.; PINHEIRO, R. S.; NORONHA, C. P.; CARVALHO, M. S. Acesso à assistência oncológica: mapeamento dos fluxos origem-destino das internações e dos atendimentos ambulatoriais. O caso do câncer de mama. Cad. Saúde Pública. 2011. Rio de Janeiro, v. $27, n^{\circ} 2$, p. 317-326, fevereiro. Disponível em: http://www.scielo.br/pdf/csp/v27n2/13.pdf . Acesso em 06 de maio 2015. https://doi.org/10.1590/S0102-311X2011000200013

PEREIRA, Anete Marília. SOARES, Beatriz Ribeiro. MONTES CLAROS E SUA REGIÃO: Novas Espacialidades, Velhos Problemas. Anais do X Encontro de Geógrafos da América Latina - 20 a 26 de março de 2005 - Universidade de São Paulo. Disponível em: http://observatoriogeograficoamericalatina.org.mx/egal10/Geografiasocioeconomica/Geografiaregional 23.pdf . Acesso em 20 de maio de 2016.

PEREIRA, A. M. Cidade Média e Região: o significado de Montes Claros no norte de Minas Gerais. Uberlândia, 2007. 351 f. Tese (Doutorado em Geografia) - Instituto de Geografia, Universidade Federal de Uberlândia, 2007.

PETERS, Sônia Helena. Avaliação da Política Nacional de Atenção Oncológica (PNAO) e o câncer de mama - dificuldade no acesso: do diagnóstico ao tratamento oncológico. 120f. Dissertação (Mestrado em Política Social). Pelotas. Universidade Católica de Pelotas - UCPEL, 2013.

RÊGO, I. K. P.; NERY, I. S. 2013. Acesso e Adesão ao Tratamento de Mulheres com Câncer de Mama Assistidas em um Hospital de Oncologia. Revista Brasileira de Cancerologia. Rio de Janeiro, v. $59, n^{\circ} 3$, p. $379-390$.

TRAVASSOS, Claúdia; CASTRO, Mônica Silva Monteiro. Determinantes e desigualdades sociais no acesso e na utilização de serviços de saúde. In: GIOVANELLA, L.; ESCOREL, S.; LOBATO, L.V.C.; NORONHA, J.C.; CARVALHO, A.I. (org.) Políticas e sistema de saúde no Brasil. - Rio de Janeiro: Editora FIOCRUZ, 2008. p. 183-206.

Recebido em: 19/02/2020

Aceito para publicação em: 23/05/2020 